\title{
New Maximal Numbers of Equilibria in Bimatrix Games*
}

\author{
B. von Stengel \\ Mathematics Department, London School of Economics, \\ London WC2A 2AE, England \\ stengel@maths.lse.ac.uk
}

\begin{abstract}
This paper presents a new lower bound of $2.414^{d} / \sqrt{d}$ on the maximal number of Nash equilibria in $d \times d$ bimatrix games, a central concept in game theory. The proof uses an equivalent formulation of the problem in terms of pairs of polytopes with $2 d$ facets in $d$-space. It refutes a recent conjecture that $2^{d}-1$ is an upper bound, which was proved for $d \leq 4$. The first counterexample is a $6 \times 6$ game with 75 equilibria. The case $d=5$ remains open. The result carries the lower bound closer to the previously known upper bound of $2.6^{d} / \sqrt{d}$.
\end{abstract}

\section{Introduction}

Consider a polytope $P$ in dimension $d$ with $2 d$ facets which is simple, that is, each vertex belongs to exactly $d$ facets of $P$. Two vertices $x$ and $y$ of $P$ form a complementary pair $(x, y)$ if every facet of $P$ is incident with $x$ or $y$. The $d$-cube has $2^{d}$ complementary vertex pairs. Is this the maximal number among the simple $d$-polytopes with $2 d$ facets? This fairly natural question seems to be open.

Here, we do not consider this problem but the following variation. Consider two simple $d$-polytopes $P$ and $Q$, each with $2 d$ facets labeled $1, \ldots, 2 d$. A vertex of a polytope has the labels of the facets it lies on. A vertex pair $(x, y)$ of $(P, Q)$ (which is a vertex of the product polytope $P \times Q$ ) is called complementary if every label $1, \ldots, 2 d$ appears as a label of $x$ or $y$. If $P$ and $Q$ are identical and identically labeled, then this is the above single-polytope problem. If $P$ and $Q$ are equal to the $d$-cube and identically labeled, then $(P, Q)$ has $2^{d}$ ordered pairs of vertices that are complementary.

However, this is not the maximal number. We will show that if $P$ and $Q$ are equal to the polar of the $d$-dimensional cyclic polytope and the labels of $Q$ are permuted relative

\footnotetext{
* This research was supported by a Heisenberg grant from the Deutsche Forschungsgemeinschaft.
} 
to the labels of $P$ in a certain way, then $(P, Q)$ has $\Theta\left((1+\sqrt{2})^{d} / \sqrt{d}\right)$ complementary vertex pairs, where $1+\sqrt{2} \approx 2.414$. Polytopes in dimension $d$ with $2 d$ facets have at most $O\left(2.6^{d} / \sqrt{d}\right)$ vertices, according to the Upper Bound Theorem for polytopes [15]. This is also the maximum number of complementary pairs since every vertex belongs to at most one such pair. Hence, there is still a gap for the maximum number, but the new lower bound of $2.414^{d} / \sqrt{d}$ offers a substantial improvement over the previously known bound of $2^{d}$.

The problem of complementary pairs for two polytopes originates from game theory in the following form: What is the maximal number of Nash equilibria of a nondegenerate $d \times d$ bimatrix game? A bimatrix game is a game for two players given by two (not necessarily square) matrices of equal dimension. The matrix entries represent the players' payoffs if player 1 chooses a row and player 2 a column as his strategy. A (Nash) equilibrium [19] is a pair of randomized strategies, one for each player, that are payoff-maximizing against each other. For each player, the upper envelope of his expected payoffs for his own strategies (against the randomized strategy of his opponent) defines a polyhedron [27], [8], [10]. After suitable projective transformation, this is a polytope, which is simple if the game is nondegenerate. For the resulting two polytopes, a complementary vertex pair corresponds to an equilibrium of the game.

In this context, the $d$-cubes arise if each player's payoff matrix is the identity matrix. Quint and Shubik [20] conjectured that these are the $d \times d$ games with a maximal number of equilibria. We refute this conjecture for $d \geq 6$ using the polytope approach. The Quint-Shubik conjecture follows for $d \leq 3$ from the Upper Bound Theorem. This has been shown for $d=4$ in [7] and [14]. The case $d=5$ is open. The single-polytope problem has no game-theoretic interpretation, not even for symmetric games, since the construction of the two polytopes (see Proposition 2.1 below) differs for the two players.

The Nash equilibrium is the central solution concept for noncooperative games [24]. Algorithms for enumerating equilibria are useful when analyzing such games. The fastest known algorithms [27], [8], [10] use vertex enumeration for polytopes, and apply even to degenerate games [29], [6]. In the games we construct here, a large number of vertices define equilibria, which shows that these algorithms cannot be substantially improved. Other algorithms for finding equilibria are surveyed in [11] and [26]. Bounds and distributions for certain kinds of equilibria are considered in [23], [12], and [13].

The correspondence between polytope pairs and equilibria of bimatrix games is explained in Section 2 (for further exposition see [25] and [26]). The construction based on cyclic polytopes is shown in Section 3. An asymptotic expression for the number of complementary vertex pairs in this class of examples is derived in Section 4.

\section{Game Equilibria and Polytopes}

We use the following notation. The transpose of a matrix $B$ is $B^{\top}$. All vectors are column vectors. The zero vector is $\mathbf{0}$, the vector of all ones is $\mathbf{1}$, their dimension depending on the context. Inequalities like $x \geq \mathbf{0}$ between two vectors hold for all components. The $n \times n$ identity matrix is $I_{n}$.

Let $(A, B)$ be a bimatrix game, where $A$ and $B$ are $m \times n$ matrices of payoffs to player 1 and player 2 , respectively. The rows are the pure strategies of player 1 and 
the columns are the pure strategies of player 2. A mixed strategy $\bar{x}$ for player 1 (or $\bar{y}$ for player 2) is a probability distribution on rows (resp. columns), written as a vector of probabilities. An equilibrium of the game is a pair $(\bar{x}, \bar{y})$ of mixed strategies so that $\bar{x}^{\top} A \bar{y} \geq x^{\top} A \bar{y}$ and $\bar{x}^{\top} B \bar{y} \geq \bar{x}^{\top} B y$ for all other mixed strategies $x$ and $y$, respectively.

In equilibrium, player 1 (and similarly player 2 ) maximizes his expected payoff $\bar{x}^{\top} A \bar{y}$ against $\bar{y}$. Equivalently [19], only those rows $i$ that have maximum payoff $u$ can have positive probability $\bar{x}_{i}$. This combinatorial condition can be expressed using the following polyhedra. Let

$$
\begin{aligned}
& \bar{P}=\left\{(\bar{x}, v) \in \mathbb{R}^{m} \times \mathbb{R} \mid \bar{x} \geq \mathbf{0}, B^{\top} \bar{x} \leq \mathbf{1} v, \mathbf{1}^{\top} \bar{x}=1\right\}, \\
& \bar{Q}=\left\{(\bar{y}, u) \in \mathbb{R}^{n} \times \mathbb{R} \mid A \bar{y} \leq \mathbf{1} u, \bar{y} \geq \mathbf{0}, \mathbf{1}^{\top} \bar{y}=1\right\} .
\end{aligned}
$$

In $\bar{Q}$, for example, the smallest value for $u$ given $\bar{y}$ defines the upper envelope of the expected payoffs for all pure strategies of player 1, given by the rows of $A \bar{y}$. In equilibrium, only optimal pure strategies $i$ may have positive probability, so that either the $i$ th inequality in $A \bar{y} \leq \mathbf{1} u$ in the definition of $\bar{Q}$ is binding ( $i$ is optimal), or the $i$ th inequality in $\bar{x} \geq \mathbf{0}$ in the definition of $\bar{P}$ is binding $\left(\bar{x}_{i}=0\right)$, or both. Similarly, a pure strategy $j$ of player 2 is optimal or not played, represented by the $j$ th inequality in $B^{\top} \bar{x} \leq \mathbf{1} v$ or in $\bar{y} \geq \mathbf{0}$ in the definition of $\bar{P}$ or $\bar{Q}$ that holds as an equality.

For identifying equilibria, it is therefore useful to consider the pure strategies of the two players as labels $1, \ldots, m+n$ numbering the $m+n$ inequalities in the definitions of $\bar{P}$ and $\bar{Q}$ in (2.1). The first $m$ of these labels represent the pure strategies of player 1 , the second $n$ those of player 2 . Then an equilibrium is a pair $(\bar{x}, \bar{y})$ so that $(\bar{x}, v) \in \bar{P}$ and $(\bar{y}, u) \in \bar{Q}$ for suitable payoffs $v$ and $u$, and for each label $1, \ldots, m+n$ the corresponding inequality in $\bar{P}$ or in $\bar{Q}$ is binding.

The polyhedra in (2.1) can be simplified by normalizing the payoffs to one and replacing probabilities by arbitrary nonnegative numbers. Let

$$
\begin{aligned}
& P=\left\{x \in \mathbb{R}^{m} \mid x \geq \mathbf{0}, B^{\top} x \leq \mathbf{1}\right\}, \\
& Q=\left\{y \in \mathbb{R}^{n} \mid A y \leq \mathbf{1}, y \geq \mathbf{0}\right\} .
\end{aligned}
$$

Then

$$
\begin{array}{rlrl}
\bar{P} & \rightarrow P, & (\bar{x}, v) & \mapsto \bar{x} / v, \\
\bar{Q} \rightarrow Q, & (\bar{y}, u) & \mapsto \bar{y} / u
\end{array}
$$

are projective transformations [30] if the payoffs $v$ and $u$ are always positive. For that purpose, we assume

$$
A \text { and } B^{\top} \text { are nonnegative and have no zero column. }
$$

This assumption can be made without loss of generality since a constant can be added to all payoffs without changing the game in a material way. We could simply assume that $A$ and $B$ are positive but want to admit examples like $A=B=I_{n}$ (if $m=n$ ) where some payoffs are zero. By (2.4), $P$ and $Q$ are polytopes (bounded polyhedra). The projective transformations (2.3) are one-to-one correspondences between $\bar{P}$ and $P-\{\mathbf{0}\}$ and $\bar{Q}$ and $Q-\{\boldsymbol{0}\}$, respectively, that preserve binding inequalities (for visualizations see [25] and [26]). The extra vertex $\mathbf{0}$ of $P$ and $Q$ arises as projection "from infinity." 
A label of a point in $P$ or $Q$ is a number in $1, \ldots, m+n$ so that the corresponding inequality in (2.2) is binding. A pair $(x, y)$ of points in $P \times Q$ is called complementary if every label $1, \ldots, m+n$ appears as a label of $x$ or of $y$. With the exception of $(\mathbf{0}, \mathbf{0})$, complementary pairs define the equilibria of the bimatrix game $(A, B)$ by renormalizing $x$ and $y$ to be vectors of probabilities.

Any complementary pair is the convex combination of extreme complementary pairs ( $x, y)$ where $x$ is a vertex of $P$ and $y$ is a vertex of $Q$ [10], [29], [6], [26]. We consider only nondegenerate (or "generic") games where only pairs of vertices can be complementary. Otherwise, the game may have infinitely many equilibria (as convex combinations of extreme equilibria). Furthermore, even the number of extreme equilibria may trivially be very large, for example if all entries of $B$ are identical (so all vertices of $P$ except $\mathbf{0}$ have all but one label) and $Q$ is a polytope with a maximum number of vertices.

A game is called nondegenerate if, against every mixed strategy $z$ of a player, there are at most $\left|\left\{i \mid z_{i}>0\right\}\right|$ pure strategies of the opponent that are optimal. This means that every point in $P$ has at most $m$ labels and every point in $Q$ has at most $n$ labels. It is easy to see that this is equivalent to the following [26]: A binding inequality for $P$ or $Q$ defines either a facet of that polytope or the empty set, but no other lower-dimensional face; and $P$ and $Q$ are simple polytopes. Inequalities that are never binding represent strictly dominated strategies [24] which are never played in equilibrium, so they can be omitted from the game. Hence, we assume that $P$ and $Q$ in (2.2) are simple polytopes with facets labeled $1, \ldots, m+n$. For complementary vertex pairs $(x, y)$, only the combinatorial structure of these polytopes matters. The special structure of the first $m$ inequalities $x \geq \mathbf{0}$ of $P$ and of the second $n$ inequalities $y \geq \mathbf{0}$ of $Q$ is not a restriction, since this can be achieved by a suitable affine transformation for each polytope, as follows.

Proposition 2.1. Let $P^{\prime}$ be a simple m-polytope and let $Q^{\prime}$ be a simple n-polytope, both with $m+n$ labeled facets, which have at least one complementary pair $\left(x^{\prime}, y^{\prime}\right)$ of vertices. Then there are $m \times n$ matrices $A$ and $B$ defining $P$ and $Q$ in (2.2), $a$ permutation of the labels $1, \ldots, m+n$ of $P^{\prime}$ and $Q^{\prime}$ yielding the labels of $P$ and $Q$, and invertible affine transformations from $P^{\prime}$ to $P$ and from $Q^{\prime}$ to $Q$ that map $\left(x^{\prime}, y^{\prime}\right)$ to $(\mathbf{0 , 0})$. Furthermore, every complementary vertex pair of $\left(P^{\prime}, Q^{\prime}\right)$ except $\left(x^{\prime}, y^{\prime}\right)$ represents a Nash equilibrium of the bimatrix game $(A, B)$.

Proof. Permute the labels $1, \ldots, m+n$ in the same way for $P^{\prime}$ and $Q^{\prime}$ such that $x^{\prime}$ has labels $1, \ldots, m$ and $y^{\prime}$ has labels $m+1, \ldots, m+n$. This does not change the complementary pairs of $\left(P^{\prime}, Q^{\prime}\right)$. Let

$$
P^{\prime}=\left\{z \in \mathbb{R}^{m} \mid C z \leq p, D z \leq q\right\}
$$

where $C z \leq p$ represents the $m$ inequalities for the facets $1, \ldots, m$ and $D z \leq q$ the remaining $n$ inequalities. For the vertex $x^{\prime}$, we have $C x^{\prime}=p$ and $D x^{\prime}<q$ since $P^{\prime}$ is simple. The $m$ binding inequalities for $x^{\prime}$ are linearly independent since $x^{\prime}$ is a vertex, so $C$ is invertible and $z \mapsto x=-C z+p$ is an affine transformation with inverse $z=-C^{-1}(x-p)$. Let $P=\left\{x \in \mathbb{R}^{m} \mid-C^{-1}(x-p) \in P^{\prime}\right\}$. Then, with $r=q-D C^{-1} p$,

$$
P=\left\{x \in \mathbb{R}^{m} \mid-x \leq \mathbf{0},-D C^{-1} x \leq r\right\} .
$$


Corresponding points of $P$ and $P^{\prime}$ have the same labels. Since the vertex $\mathbf{0}$ of $P$ corresponds to $x^{\prime}$ in $P^{\prime}, \mathbf{0}<r$. Thus, the $j$ th row of $-D C^{-1} x \leq r$ can be normalized by multiplication with the scalar $1 / r_{j}$, so we can assume $r=1$. Then $P$ is defined as in (2.2) with the $n \times m$ transposed payoff matrix $B^{\top}=-D C^{-1}$. Similarly, we can find an $m \times n$ matrix $A$ so that $Q$ in (2.2) is an affine transform of $Q^{\prime}$. The complementary vertex pairs of $\left(P^{\prime}, Q^{\prime}\right)$ except $\left(x^{\prime}, y^{\prime}\right)$ correspond to the Nash equilibria of $(A, B)$ by construction. If desired, a constant can be to the entries of $A$ and $B$ to obtain (2.4), which does not change the combinatorial structure of $P$ and $Q$.

Polytopes $P^{\prime}$ and $Q^{\prime}$ with general labeling may have no complementary pairs at all, so this case is explicitly excluded in Proposition 2.1. Interestingly, the number of complementary pairs of $(P, Q)$ in Proposition 2.1 is always even, since the algorithm by Lemke and Howson [9], [21], [28] connects complementary pairs in $P \times Q$ by paths where a given label is missing. It computes one Nash equilibrium of the game when started from $(\mathbf{0}, \mathbf{0})$.

\section{Cyclic Polytopes and a Lower Bound}

We specialize the problem of finding nondegenerate $m \times n$ games with a large number of equilibria to square games where $m=n=d$. Then (2.2) defines two simple $d$ polytopes $P$ and $Q$ with $2 d$ facets. For the $d \times d$ game with $A=B=I_{d}$, both polytopes $P$ and $Q$ are equal to the $d$-cube $[0,1]^{d}$ which has $2^{d}$ vertices, each of which is part of a complementary pair. The Quint-Shubik conjecture [20] states that this is the maximum number.

We refute this conjecture for $d \geq 6$. Any counterexample yields a counterexample in higher dimensions, as follows. If the polytope pair $(P, Q)$ in dimension $d$ has $E$ complementary pairs, then $\left(P^{\prime}, Q^{\prime}\right)$ with $P^{\prime}=P \times[0,1]$ and $Q^{\prime}=Q \times[0,1]$ is a polytope pair in dimension $d+1$ with $2 E$ complementary pairs. Namely,

$$
\begin{aligned}
& P^{\prime}=\left\{\left(x, x_{d+1}\right) \in \mathbb{R}^{d} \times \mathbb{R} \mid x \geq \mathbf{0}, x_{d+1} \geq 0, B^{\top} x \leq \mathbf{1}, x_{d+1} \leq 1\right\}, \\
& Q^{\prime}=\left\{\left(y, y_{d+1}\right) \in \mathbb{R}^{d} \times \mathbb{R} \mid A y \leq \mathbf{1}, y_{d+1} \leq 1, y \geq \mathbf{0}, y_{d+1} \geq 0\right\},
\end{aligned}
$$

so that any complementary vertex pair $(x, y)$ of $(P, Q)$ yields the two complementary pairs $((x, 0),(y, 0))$ and $((x, 1),(y, 1))$ of $\left(P^{\prime}, Q^{\prime}\right)$.

Our counterexamples are based on the polars of cyclic polytopes, which have a maximum number of vertices. The polar [30] of a polytope $P$ that is the convex hull of the $d$-vectors $c_{1}, \ldots, c_{N}$ is given by

$$
P^{\Delta}=\left\{x \in \mathbb{R}^{d} \mid c_{i}^{\top} x \leq 1,1 \leq i \leq N\right\},
$$

provided $P$ (and then also $P^{\Delta}$ ) has $\mathbf{0}$ in its interior, which can always be achieved by translating $P$. Any face of $P^{\Delta}$ of dimension $d-k$ is defined by $k$ binding inequalities in (3.2), and corresponds to a face of dimension $k-1$ of $P$, given by the convex hull of the corresponding $k$ vertices of $P$.

A cyclic polytope $C_{d}(N)$ [3], [30] in dimension $d$ with $N$ vertices is defined as the convex hull of any $N$ points on the moment curve $\{\mu(t) \mid t \in \mathbb{R}\}$ in $\mathbb{R}^{d}, \mu(t)=$ 
$\left(t, t^{2}, \ldots, t^{d}\right)^{\top}$. Any $d+1$ points on this curve are affinely independent, so $C_{d}(N)$ is simplicial (no facet contains more than $d$ vertices). The particular choice of the points $\mu\left(t_{1}\right), \ldots, \mu\left(t_{N}\right)$ on the moment curve does not affect the combinatorial structure of $C_{d}(N)$. Assume $t_{1}<\cdots<t_{N}$. A set $S$ of $d$ vertices corresponds to a $0-1$ string $s=s_{1} s_{2} \cdots s_{N}$ with $s_{i}=1$ if $\mu\left(t_{i}\right) \in S$ and $s_{i}=0$ otherwise. The hyperplane $H$ through the points in $S$ defines a facet of $C_{d}(N)$ if and only if the string $s$ fulfills the Gale evenness condition [3], that is, it contains no substring $s_{i} \cdots s_{j}=01 \cdots 10$ with an odd number $i-j-1$ of 1's (like 01110). Otherwise, the two vertices $\mu\left(t_{i}\right)$ and $\mu\left(t_{j}\right)$ would be on opposite sides of $H$, since the moment curve changes from one side of $H$ to the other at the points $\mu\left(t_{i}\right), i \in S$. The Gale evenness condition is symmetric with respect to a cyclic shift of the string $s$ if $d$ is even.

The number $\Phi(d, N)$ of facets of $C_{d}(N)$ is the number of the $0-1$ strings $s$ fulfilling the Gale evenness condition. If $d$ is even, $d=2 l$, then either $s$ starts and ends with an even number of 1's, and is composed of $l$ substrings 11 and $N-d 0$ 's, or $s$ is such a string with $l-1$ substrings 11 and an additional 1 put at each end. Hence,

$$
\Phi(2 l, N)=\left(\begin{array}{c}
N-l \\
l
\end{array}\right)+\left(\begin{array}{c}
N-l-1 \\
l-1
\end{array}\right)=\frac{N}{l}\left(\begin{array}{c}
N-l-1 \\
l-1
\end{array}\right) .
$$

Similarly, one can show

$$
\Phi(2 l+1, N)=2\left(\begin{array}{c}
N-l-1 \\
l
\end{array}\right) .
$$

No $d$-polytope with $N$ vertices has more facets than the cyclic polytope $C_{d}(N)$, according to the Upper Bound Theorem for polytopes [15], [18]. Applied to the polars, this implies that no $d$-polytope with $N$ facets has more than $\Phi(d, N)$ vertices. Hence, the polytopes $P$ and $Q$ in (2.2) have at most $\Phi(m, m+n)$ and $\Phi(n, m+n)$ vertices, respectively. The bound is stricter for the polytope of smaller dimension since $\Phi(d, N)<\Phi(d+1, N)$ if $d<N / 2$. This implies the following bound on the number of equilibria [7]:

Proposition 3.1. A nondegenerate $m \times n$ bimatrix game, $m \leq n$, has at most $\Phi(m, m+$ n) - 1 Nash equilibria.

For $m=n, \Phi(n, 2 n)$ grows asymptotically from $n$ to $n+1$ by an average factor of $\sqrt{27 / 4}=2.598 \ldots$, much faster than $2^{n}$. We consider more precise asymptotics in Section 4 .

In our construction, we let $P=Q=C_{d}(2 d)^{\Delta}$, which are simple polytopes since $C_{d}(2 d)$ is simplicial. We consider only even dimensions $d$. In odd dimension, the polytopes $P^{\prime}$ and $Q^{\prime}$ in (3.1) constructed from $P$ and $Q$ in the next lower even dimension $d$ have a larger number of complementary vertex pairs than those based on the cyclic polytopes in dimension $d+1$.

So, in the following, observe that $d=2 l$ is even, there are $N=2 d=4 l$ points on the moment curve, whose convex hull defines $C_{d}(N)$, and which, after translation so that $\mathbf{0}$ is in the interior of that polytope, represent the normal vectors of the facets of its polar $C_{d}(N)^{\Delta}$ as in (3.2), which have to be suitably labeled. Furthermore, the facets of $C_{d}(N)$ define the vertices of $P$ and $Q$, among which we look for complementary pairs. 
It suffices to look at the representation of these vertices of $P$ and $Q$, the facets of $C_{d}(N)$, by $0-1$ strings $s=s_{1} s_{2} \cdots s_{N}$, for example $s=01101100$ if $d=4, N=8$. These strings are balanced, that is, contain the same number of 0's and 1's, and fulfill the Gale evenness condition. We can assume that the labeling of the $N$ facets of $P$ is in the order of the positions in this string. The labeling of the facets of $Q$ is given by a certain permutation $v$ of $\{1, \ldots, N\}$, such that $s$ defining a vertex of $P$ is part of a complementary pair if and only if the complementary permuted string

$$
\bar{s}_{\nu}:=\bar{s}_{\nu(1)} \bar{s}_{\nu(2)} \cdots \bar{s}_{\nu(N)}
$$

defines a vertex of $Q$, that is, fulfills the Gale evenness condition, where $\overline{0}=1$ and $\overline{1}=0$. For example, suppose that $v$ is the identity permutation. Then for $s=01101100$, $\bar{s}_{v}=10010011$ which does not fulfill Gale evenness, whereas $\bar{s}_{\nu}$ does for $s=00011110$. For these two strings $s$, the opposite holds when considering the permutation

$$
v(i)=\left\{\begin{array}{ll}
i-1 & \text { if } i \text { is even, } \\
i+1 & \text { if } i \text { is odd, }
\end{array} \quad 1 \leq i \leq N .\right.
$$

With the identity permutation $v$, the polytopes $P$ and $Q$ do not have more than $2^{d}$ complementary vertex pairs, since only the strings $s$ that are composed of substrings 00 and 11, except at the ends, have the property that both $s$ and $\bar{s}_{v}$ fulfill Gale evenness. However, the permutation $v$ in (3.4) leads to a number of complementary vertex pairs that exceeds $2^{d}$ for all even $d \geq 6$.

Proposition 3.2. Let $S(l)$ be the set of balanced $0-1$ strings of length $4 l$ composed of the substrings 00, 11, and 0110. Let $s$ be any balanced $0-1$ string of length $N=2 d$, and let $d=2 l$. Then for the permutation $v$ in (3.4), $s$ and $\bar{s}_{v}$ fulfill the Gale evenness condition if and only if $s \in S(l)$ or $s=10 s^{\prime} 01$ for some $s^{\prime} \in S(l-1)$.

Proof. Clearly, any string $s$ as described fulfills the Gale evenness condition. The substrings 00,11 , and 0110 in $s$ are complemented to 11,00 , and 1001, respectively, and permuted by $v$ to substrings 11,00 , and 0110 , respectively, in $\bar{s}_{v}$. Similarly, an initial or terminal substring 10 or 01 is left as it is, so $\bar{s}_{\nu}$ also fulfills Gale evenness. Conversely, suppose $s$ is not of the described form. If $s$ starts with the substring 10, remove it. Then remove repeatedly all initial substrings 00,11 , or 0110 from $s$. If the remainder starts with 10, 0100, or 0101, the Gale evenness condition fails for $s$. If it starts with 0111 (the only possibility left), it becomes 0100 in $\bar{s}_{v}$ so the condition fails there.

Let $E(d)$ be the number of complementary vertex pairs of $(P, Q)$ in our construction, where $P=Q=C_{d}(2 d)^{\Delta}$ and the labels of $Q$ are permuted by $v$. By Proposition 3.2, $E(d)$ is determined by the number $\sigma(l):=|S(l)|$ of balanced 00-11-0110 strings of length $4 l=2 d$, namely

$$
E(2 l)=\sigma(l)+\sigma(l-1) .
$$

Proposition 3.2 can be extended to odd dimension showing $E(2 l+1)=2 \sigma(l)$, but, as mentioned, this number is smaller than $2 \cdot E(2 l)$ for the polytopes $\left(P^{\prime}, Q^{\prime}\right)$ constructed in (3.1). 
If a string in $S(l)$ contains $k$ substrings $00,0 \leq k \leq l$, then it contains the same number of substrings 11 since it is balanced, and $l-k$ substrings 0110 . These substrings may be arranged in any manner, with $(l+k) ! /(k ! k !(l-k) !)$ many possibilities. Hence,

$$
\sigma(l)=\sum_{k=0}^{l} \frac{(l+k) !}{k ! k !(l-k) !}=\sum_{k=0}^{l}\left(\begin{array}{c}
l+k \\
k
\end{array}\right)\left(\begin{array}{l}
l \\
k
\end{array}\right)
$$

The first values of $\sigma(l)$ are given as follows. The numbers $\tilde{\sigma}(l)$ are an asymptotic approximation that we prove in the next section.

\begin{tabular}{l|ccccccccc}
$l$ & 0 & 1 & 2 & 3 & 4 & 5 & 6 & 7 & 8 \\
\hline$\sigma(l)$ & 1 & 3 & 13 & 63 & 321 & 1683 & 8989 & 48,639 & 265,729 \\
$\tilde{\sigma}(l)$ & & 3.4 & 13.8 & 65.5 & 330.4 & 1722.6 & 9165.3 & $49,456.6$ & $269,636.8$
\end{tabular}

Our construction produces the first counterexample to the Quint-Shubik conjecture for $d=6$ since $E(6)=76>2^{6}$ (already $E(4)=16=2^{4}$, where the equilibria are quite different from the game where $A=B=I_{4}$ ). A specific $6 \times 6$ bimatrix game with 75 Nash equilibria using the points $\mu(t)$ and $\mu(-t)$ for $t=1,2, \ldots, 6$ on the moment curve, a translation so that the barycenter of these points is $\mathbf{0}$ (for polarity), and Proposition 2.1, is described in [25].

Does the permutation (3.4) yield the maximum number of complementary pairs for the cyclic polytopes? Trying out all $(2 d)$ ! permutations shows that it does for $d=6$, where the permutation is unique up to the symmetry (cyclic shift and reversal) of the strings fulfilling the Gale evenness condition. This computation takes hours for $d=6$ and therefore was not attempted for $d=8$. We sketch a proof that (3.4) is optimal for $d=6$. Any Gale evenness string not in $S(3)$ has a substring 011110 or 01111110 starting on an even position. For example, it may be $011110 s$ with $s=001100$. Consider the strings $011011 s$ and $001111 s$ which belong to $S(3)$ and which both differ from $011110 s$ in only one label. Hence, these are vertices of $P$ forming a triangle or "clique" [14]. The complementary sets of labels for these vertices share $d-1$ labels. The corresponding $d-1$ facets of $Q$ meet in an edge (if at all) that contains only two vertices of $Q$. Hence, a face of $P$ that is a triangle can have at most two vertices that are part of complementary pairs, and the two in $S(3)$ already are, so 011110 s is not unless another complementary pair is sacrificed. Similarly, replacing a substring 01111110 by its neighbors 01111011 , 01101111 , and 00111111 defines a simplex as a face of $P$ with again only two vertices that can be part of complementary pairs. So the 00-11-0110 strings indeed yield the maximum number of complementary pairs for $d=6$. It may be interesting to extend this argument to higher dimensions.

The question for general polytopes remains open, for $d \geq 5$. For $d=4$, the $d$ polytopes characterized in [5] and their triangles show that no polytope pair has more complementary vertex pairs than the cubes [7]. 


\section{Asymptotics of Upper and Lower Bounds}

In dimension $d$, the maximal number of complementary vertex pairs is bounded from above by $\Phi(d, 2 d)$ by the Upper Bound Theorem and from below by $E(d)$ as defined by (3.5) and (3.6). In order to compare these functions better with $2^{d}$, we will find asymptotically equal expressions. Functions $f, g$ are called asymptotically equal, denoted $f(n) \sim g(n)$ as $n \rightarrow \infty$, if $f(n) / g(n) \rightarrow 1$, that is, the relative error goes to zero [4]. We apply Stirling's formula

$$
n ! \sim \sqrt{2 \pi n}\left(\frac{n}{e}\right)^{n}
$$

to the upper bound $U(d):=\Phi(d, 2 d)$ in (3.3) for even $d$, which yields

$$
U(2 l)=2\left(\begin{array}{c}
3 l-1 \\
l
\end{array}\right)=2 \frac{2 l}{3 l}\left(\begin{array}{c}
3 l \\
l
\end{array}\right) \sim \frac{2}{3} \sqrt{\frac{3}{\pi l}}\left(\frac{27}{4}\right)^{l}
$$

or

$$
U(d) \sim 2 \sqrt{\frac{2}{3 \pi}} \frac{\sqrt{27 / 4}^{d}}{\sqrt{d}} \approx 0.921 \frac{2.5981^{d}}{\sqrt{d}}
$$

for even $d$, where 0.921 is replaced by $\sqrt{2 / \pi} \approx 0.798$ if $d$ is odd.

Finding a similar asymptotic expression for $\sigma(l)$ in (3.6) is more interesting. This integer sequence has been studied before, as (3.7) looked up in [22] (and its electronic server, described there) reveal. The number $\sigma(n)$ is the number of "King paths on a chessboard" [16], that is, the number of paths in a two-dimensional integer lattice from $(0,0)$ to $(n, n)$ where the allowed steps are one unit right, up, or diagonal (each such step corresponding to a substring 00,11 , or 0110 , respectively). According to [1, p. 81], $\sigma(n)=P_{n}(3)$ for the $n$th Legendre polynomial $P_{n}$ defined explicitly by

$$
P_{n}(x)=\sum_{k=0}^{n}\left(\begin{array}{c}
n+k \\
k
\end{array}\right)\left(\begin{array}{l}
n \\
k
\end{array}\right)\left(\frac{x-1}{2}\right)^{k}
$$

[17] or recursively by $P_{0}(x)=1, P_{1}(x)=x$, and

$$
P_{n}(x)=x\left(2-\frac{1}{n}\right) P_{n-1}(x)-\left(1-\frac{1}{n}\right) P_{n-2}(x) .
$$

The recurrence (4.3) can be verified by (4.2). For $x=3, \sigma(n)=P_{n}(3)$, it can-with some effort-also be given a combinatorial interpretation in terms of the lattice paths with diagonal steps. Using the generating function

$$
g(y)=\sum_{n \geq 0} \sigma(n) y^{n},
$$

the recurrence (4.3) for $x=3$ is equivalent to the differential equation

$$
g^{\prime}(y)\left(1-6 y+y^{2}\right)+g(y)(y-3)=0
$$


which, with $g(0)=\sigma(0)=1$, has the unique solution

$$
g(y)=\frac{1}{\sqrt{1-6 y+y^{2}}} .
$$

Regarded as a function on the complex plane $\mathbb{C}$, the function $g$ is analytic around the origin with Taylor coefficients $\sigma(n)$ as in (4.4). We use a theorem of [2] that shows how to obtain information about these coefficients from the behavior of $g$ at its dominant singularity (the one with smallest absolute value). For simplicity, we state this theorem with overly strong assumptions concerning the domain $\Delta$ of the function which we define here as

$$
\Delta=\mathbb{C}-\{z \in \mathbb{R} \mid z \geq 1\}
$$

Theorem 4.1 [2, Corollary 2]. Assume that $f(z)$ is analytic in $\Delta$ and that, as $z \rightarrow 1$ in $\Delta$,

$$
f(z) \sim K(1-z)^{\alpha},
$$

where $K$ and $\alpha$ are real constants, $\alpha$ not a nonnegative integer. Then, as $n \rightarrow \infty$,

$$
\left[z^{n}\right] f(z) \sim \frac{K}{\Gamma(-\alpha)} n^{-\alpha-1} .
$$

In (4.6), $\left[z^{n}\right] f(z)$ is the Taylor coefficient of $z^{n}$ in the expansion of $f(z)$, and $\Gamma$ is the Gamma function, where $\Gamma\left(\frac{1}{2}\right)=\sqrt{\pi}$. We use Theorem 4.1 for $\alpha=-\frac{1}{2}$ but have to normalize the dominant singularity of $g(y)$ to one. It is given by the smaller root $r$ of the roots $r$ and $R$ of the polynomial $1-6 y+y^{2}$,

$$
r=3-2 \sqrt{2}, \quad R=3+2 \sqrt{2},
$$

so that

$$
g(y)=((r-y)(R-y))^{-1 / 2}=\left(r(R-y)\left(1-\frac{y}{r}\right)\right)^{-1 / 2} .
$$

Let $z=y / r, y=r z$, so that $g(r z)$ is defined for $z \in \Delta$ and

$$
f(z)=g(r z)=(r(R-r z)(1-z))^{-1 / 2} \sim(r(R-r))^{-1 / 2}(1-z)^{-1 / 2}
$$

as $z \rightarrow 1$. Using $1 / r=R=(1+\sqrt{2})^{2},(4.6)$ yields

$$
\left[z^{n}\right] f(z) \sim \frac{1+\sqrt{2}}{2^{5 / 4} \sqrt{\pi n}}
$$

as $n \rightarrow \infty$ and, since $g(y)=f(y / r)$,

$$
\sigma(n) \sim \tilde{\sigma}(n):=\frac{1+\sqrt{2}}{2^{5 / 4} \sqrt{\pi}} \frac{(1+\sqrt{2})^{2 n}}{\sqrt{n}} .
$$

The relative error of this approximation is for $n \geq 6$ less than $2 \%$ as (3.7) shows. A better approximation [2] would introduce factors like $(1+c / n)$ for a constant $c$ so 
that the relative error is of order $O\left(n^{-2}\right)$ rather than $O\left(n^{-1}\right)$, as is known for Stirling's formula [4]. We have not investigated this further.

The asymptotic expression becomes simpler when used for the number $E(d)$ of complementary vertex pairs in (3.5) since $1+\sqrt{2}$ appears in the denominator and cancels. Expressed in terms of $d$, as $d \rightarrow \infty$,

$$
E(d) \sim \frac{2^{3 / 4}}{\sqrt{\pi}} \frac{(1+\sqrt{2})^{d}}{\sqrt{d}} \approx 0.949 \frac{2.414^{d}}{\sqrt{d}},
$$

if $d$ is even, with 0.949 replaced by $\left(2^{9 / 4}-2^{7 / 4}\right) / \sqrt{\pi} \approx 0.786$ if $d$ is odd, using the construction in (3.1).

As (4.1) and (4.7) show, the number $E(d)$ of complementary vertex pairs in our construction is not that far away from the upper bound $U(d)$, at least compared with the previously known lower bound $2^{d}$. We summarize our result, where the upper bound is due to [7].

Theorem 4.2. The maximal number of Nash equilibria in a nondegenerate $d \times d$ bimatrix game is bounded from above by $U(d)-1$ and from below by $E(d)-1$. As $d \rightarrow \infty$, asymptotic expressions for $U(d)$ and $E(d)$ for even $d$ are (4.1) and (4.7), respectively.

\section{Acknowledgments}

The author thanks Nicola Galli, Gyula Karolyi, Andrew McLennan, Raimund Seidel, Emo Welzl, Günter Ziegler, and the referees for helpful comments and discussions.

\section{References}

1. L. Comtet, Advanced Combinatorics, Reidel, Dordrecht, 1974.

2. P. Flajolet and A. Odlyzko, Singularity analysis of generating functions, SIAM J. Discrete Math. 3 (1990), 216-240.

3. D. Gale, Neighborly and cyclic polytopes, in Convexity, pp. 225-232, V. Klee (ed.), Proc. Symposia in Pure Mathematics, Vol. 7, American Mathematical Society, Providence, RI, 1963.

4. R. L. Graham, D. E. Knuth, and O. Patashnik, Concrete Mathematics, Addison-Wesley, Reading, MA, 1991.

5. B. Grünbaum and V. P. Sreedharan, An enumeration of simplicial 4-polytopes with 8 vertices, J. Combin. Theory 2 (1967), 437-465.

6. M. J. M. Jansen, Maximal Nash subsets for bimatrix games, Naval Res. Logist. Quart. 28 (1981), 147-152.

7. H. Keiding, On the maximal number of Nash equilibria in an $n \times n$ bimatrix game, Games Econom. Behavior 21, 1997, 148-160.

8. H. W. Kuhn, An algorithm for equilibrium points in bimatrix games, Proc. Nat. Acad. Sci. U.S.A. 47 (1961), 1657-1662.

9. C. E. Lemke and J. T. Howson, Jr., Equilibrium points of bimatrix games, J. Soc. Indust. Appl. Math. 12 (1964), 413-423.

10. O. L. Mangasarian, Equilibrium points of bimatrix games, J. Soc. Indust. Appl. Math. 12 (1964), 778-780.

11. R. D. McKelvey and A. McLennan, Computation of equilibria in finite games, in Handbook of Computational Economics, Vol. I, pp. 87-142, H. M. Amman, D. A. Kendrick, and J. Rust (eds.), Elsevier, Amsterdam, 1996. 
12. R. D. McKelvey and A. McLennan, The maximal number of regular totally mixed Nash equilibria, J. Econom. Theory 72 (1997), 411-425.

13. A. McLennan, The maximal generic number of pure Nash equilibria, J. Econom. Theory 72 (1997), 408-410.

14. A. McLennan and I.-U. Park, Generic $4 \times 4$ two person games have at most 15 Nash equilibria, Games Econom. Behavior, to appear.

15. P. McMullen, The maximum number of faces of a convex polytope, Mathematika 17 (1970), 179-184.

16. L. Moser, King paths on a chessboard, Math. Gaz. 39 (1955), 54.

17. L. Moser and W. Zayachkowski, Lattice paths with diagonal steps, Scripta Math. 26 (1961), 223-229.

18. K. Mulmuley, Computational Geometry: An Introduction Through Randomized Algorithms, Prentice-Hall, Englewood Cliffs, NJ, 1994.

19. J. F. Nash, Non-cooperative games. Ann. of Math. 54 (1951), 286-295.

20. T. Quint and M. Shubik, A theorem on the number of Nash equilibria in a bimatrix game, Internat. J. Game Theory 26 (1997), 353-360.

21. L. S. Shapley, A note on the Lemke-Howson algorithm, Math. Programming Stud. 1 (1974), 175-189.

22. N. J. A. Sloane and S. Plouffe, The Encyclopedia of Integer Sequences, Academic Press, San Diego, CA, 1995.

23. W. Stanford, The limit distribution of pure strategy Nash equilibria in symmetric bimatrix games, Math. Oper. Res. 21 (1996), 726-733.

24. E. van Damme, Stability and Perfection of Nash Equilibria, Springer-Verlag, Berlin, 1987.

25. B. von Stengel, New lower bounds for the number of equilibria in bimatrix games, Technical Report 264, Dept. of Computer Science, ETH Zürich, 1997.

26. B. von Stengel, Computing equilibria for two-person games, in Handbook of Game Theory, Vol. 3, R. J. Aumann and S. Hart (eds.), North-Holland, Amsterdam, to appear.

27. N. N. Vorob'ev, Equilibrium points in bimatrix games, Theory Probab. Appl. 3 (1958), 297-309.

28. R. Wilson, Computing simply stable equilibria, Econometrica 60 (1992), 1039-1070.

29. H.-M. Winkels, An algorithm to determine all equilibrium points of a bimatrix game, in Game Theory and Mathematical Economics, pp. 137-148, O. Moeschlin and D. Pallaschke (eds.), North-Holland, Amsterdam, 1979.

30. G. M. Ziegler, Lectures on Polytopes, Springer-Verlag, New York, 1995.

Received July 23, 1997, and in revised form June 26, 1998. 